\title{
APP intracellular domain acts as a transcriptional regulator of miR-663 suppressing neuronal differentiation
}

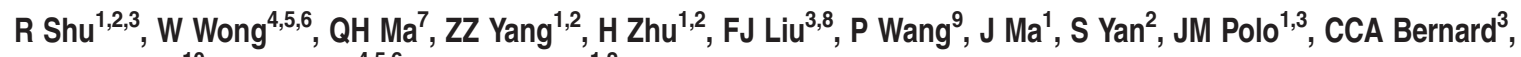 \\ LW Stanton ${ }^{\star, 10}$, GS Dawe ${ }^{*, 4,5,6}$ and ZC Xiao ${ }^{*, 1,2}$
}

Amyloid precursor protein (APP) is best known for its involvement in the pathogenesis of Alzheimer's disease. We have previously demonstrated that APP intracellular domain (AICD) regulates neurogenesis; however, the mechanisms underlying AICD-mediated regulation of neuronal differentiation are not yet fully characterized. Using genome-wide chromatin immunoprecipitation approaches, we found that AICD is specifically recruited to the regulatory regions of several microRNA genes, and acts as a transcriptional regulator for miR-663, miR-3648 and miR-3687 in human neural stem cells. Functional assays show that AICD negatively modulates neuronal differentiation through miR-663, a primate-specific microRNA. Microarray data further demonstrate that miR-663 suppresses the expression of multiple genes implicated in neurogenesis, including FBXL18 and CDK6. Our results indicate that AICD has a novel role in suppression of neuronal differentiation via transcriptional regulation of miR-663 in human neural stem cells.

Cell Death and Disease (2015) 6, e1651; doi:10.1038/cddis.2015.10; published online 19 February 2015

Amyloid precursor protein (APP), a ubiquitous type I transmembrane receptor, is processed by $\alpha$-, $\beta$ - and $\gamma$-secretase enzymes in two distinct cascades. Processing of APP by $\beta$ - and $\gamma$-secretase in the amyloidogenic cascade leads to release of amyloid beta peptide $(A \beta)$, while in the nonamyloidogenic pathway, mediated by $\alpha$ - and $\gamma$-secretase, $A \beta$ is not produced. The biological role of $A \beta$ has been the focus of considerable research interest as it is associated with the formation of amyloid plaques in the pathology of Alzheimer's disease (AD). ${ }^{1}$ However, the function of the APP intracellular domain (AICD), another APP-derived cleavage product, remains incompletely understood. ${ }^{2}$ Over the past decades, AICD has sparked research interest for its roles in apoptosis, synaptic plasticity and neural development.. ${ }^{3,4}$ In addition, AICD transgenic or knock-in mutant animal models have been reported to display $A D$-like pathological features, such as neuronal loss, tau aggregation, neuroinflammation, impaired neurogenesis and cognitive performance..$^{5-10}$

MicroRNAs (miRNAs) are widely distributed, small, noncoding RNA molecules that have emerged as post- transcriptional regulators of genes involved in developmental processes and disease. ${ }^{11}$ In the nervous system, some miRNAs act as key post-transcriptional regulators in neurogenesis, axonal pathfinding, apoptosis and synaptic plasticity. ${ }^{12,13}$ Moreover, several miRNA-profiling studies have shown that miRNA expression patterns are altered in $A D$ brains and peripheral tissues. However, whether the changes of miRNA pattern are the cause or the consequence of the disease remains elusive. ${ }^{14}$

We have previously shown that transient axonal glycoprotein-1 interacts with APP as a novel ligand, and this interaction results in the inhibition of neurogenesis through an AICD-mediated action. ${ }^{15,16}$ Upon processing of APP, AICD is released and translocates into the nucleus. Once AICD is in the nucleus, it can influence gene transcription. ${ }^{17,18} \mathrm{~A}$ recent in vivo study showed that APP could regulate neurogenesis by antagonizing miR-574-5p in the developing cerebral cortex of mice. ${ }^{19}$ However, the molecular mechanism by which APP inhibits neural stem cell (NSC) differentiation remains to be determined.

\footnotetext{
${ }^{1}$ Shunxi-Monash Immune Regeneration and Neuroscience Laboratories, Department of Anatomy and Developmental Biology, Monash University, Clayton, Victoria 3800, Australia; ${ }^{2}$ The Key Laboratory of Stem Cell and Regenerative Medicine, Institute of Molecular and Clinical Medicine, Kunming Medical College, Kunming 650031, China; ${ }^{3}$ Australian Regenerative Medicine Institute, Monash University, Clayton, Victoria 3800, Australia; ${ }^{4}$ Department of Pharmacology, Yong Loo Lin School of Medicine, National University Health System, National University of Singapore, Singapore 119077, Singapore; ${ }^{5}$ Neurobiology and Ageing Programme, Life Sciences Institute, National University of Singapore, Singapore 119077, Singapore; ${ }^{6}$ Singapore Institute for Neurotechnology (SINAPSE), Singapore 117456, Singapore; ${ }^{7}$ Institute of Neuroscience, Soochow University, Suzhou 215123, China; ${ }^{8}$ College of Animal Science and Technology, Henan University of Science and Technology, Luoyang 471000, China; ${ }^{9}$ Department of Pediatrics, Duke University School of Medicine, Durham, NC 27710, USA and ${ }^{10}$ Stem Cell and Developmental Biology Group, Genome Institute of Singapore, Singapore 138672, Singapore

${ }^{*}$ Corresponding authors: ZC Xiao or GS Dawe or LW Stanton, Shunxi-Monash Immune Regeneration and Neuroscience Laboratories, Department of Anatomy and Developmental Biology, Monash University, Clayton, Victoria 3800, Australia. Tel: +61 39902 4574; Fax: +61 39905 0680; E-mail: zhicheng.xiao@monash.edu or gavindawe@nus.edu.sg or stantonl@gis.a-star.edu.sg

Abbreviations: APP, Amyloid precursor protein; A $\beta$, amyloid beta peptide; AD, Alzheimer's disease; AICD, APP intracellular domain; miRNA, microRNA; NSC, neural stem cell; ChIP-seq, chromatin immunoprecipitation coupled with deep DNA sequencing; TTS, transcription terminal site; TSS, promoter-transcription start site; SSS, stemloop start sites; FAC, functional annotation clustering; ENCODE, Encyclopedia of DNA Elements; UTR, untranslated region; pri-miRNA, primary miRNA; SCF, SKP1-CUL1F-box protein; LFC, log2-transformed gene expression fold changes; CDF, cumulative distribution function; qPCR, quantitative PCR

Received 14.11.14; accepted 29.12.14; Edited by A Verkhratsky
} 
In this study, we hypothesized that APP might influence physiological processes, such as neurogenesis, via direct binding of AICD to the miRNA-embedding genomic region. To test this hypothesis, we applied a genome-wide search for AICD-regulated miRNAs, using chromatin immunoprecipitation coupled with deep DNA sequencing (ChIP-seq), and then selected dozens of candidate miRNAs to validate their regulation by AICD as well as their role in the neuronal differentiation of human neural stem cells (hNSCs). Our findings demonstrate that AICD binds to regulatory regions of specific miRNAs in human genome and suppresses neuronal differentiation through transcriptional regulation of miR-663.

\section{Results}

Distribution of the AICD ChIP-seq miRNA-binding peaks. In the genome, miRNAs are located either between independent transcription units (intergenic), or in the intronic or exonic regions of genes. The intergenic miRNAs are transcribed independently, whereas the intronic and exonic miRNAs may be transcribed with their host genes. To comprehensively identify AICD-binding sites within the promoter regions of miRNAs, duplicate ChIP-seq experiments were performed in SH-SY5Y cells. The AICD-binding sites generated from the two data sets were mapped on the genome relative to the nearest miRNAs and annotated with respect to their distance from the miRNA stem-loop start sites (SSS; Supplementary Tables S1 and S2). Analysis of the pooled data showed that AICD binds to 576 sites corresponding to 304 miRNAs in set 1 , and 478 sites corresponding to 263 miRNAs in set 2 , with an overlap of 207 miRNAs (Figures 1a-c). These results suggest that the binding between AICD and miRNA regions is highly reproducible through the ChIP-seq assays. Notably, most reported AICD-regulated genes were also found in our ChIPseq data, representing robust controls for verifying the reliability of our ChIP-seq data (Supplementary Table S3).

Genome analysis of the ChIP-seq data shows that most AICD-binding sites for miRNAs are enriched in intergenic and intronic regions, but not in the promoter regions of proteincoding genes (Figures $1 \mathrm{a}$ and $\mathrm{b}$ ), as most of the miRNAs are located in the intergenic or intronic regions. ${ }^{20}$ As we know, nearly all the intergenic miRNAs and $\sim 35 \%$ of the intronic miRNAs, which consist of the majority of miRNAs, have their own promoters, ${ }^{21,22}$ whereas the ChIP-seq-binding sites in our study are not randomly distributed but are enriched in the proximal miRNA SSS (Figures 1d and e). Taken together, our data indicate that $A I C D$ binds to the upstream regulatory elements of these miRNAs.

Functional annotation of the AICD-regulated miRNAs. Within the 207 miRNAs identified in the two ChIP-seq data sets, 93 miRNAs are associated with 1471 validated target genes according to the miRWalk database (Supplementary Table S4). ${ }^{23}$ Meanwhile, 93 randomly selected miRNAs, which were not in our ChIP-seq data sets, were used as controls. Using functional annotation clustering (FAC), we compared the enrichment of gene ontology terms between the AICD and control miRNA group. ${ }^{24}$ The outcome suggests that the best-known functions of AICD are enriched in the AICD-regulated miRNAs (Figure $1 f$ and Supplementary Table S5). Therefore, we hypothesized that AICD may perform its roles through regulating miRNAs. To test this hypothesis, these miRNAs should be validated step-by-step through ChIP, miRNA expression and functional assays, as well as target gene identification.

Validation of selected AICD-regulated miRNAs using ChIP. To select high-priority candidates, the miRNAs from the two ChIP-seq data sets were ranked by 'peak score' (Figures 2a and b; Supplementary Tables S1and S2). From the top 20 binding sites of the two sets (R1: Figure 2a; R2: Figure 2b), 12 miRNAs were found in common and were selected for further evaluation (Figure $2 c$ and Table 1). Given that the regulation of gene expression through AICD is affected by the chromatin status of cell types, ${ }^{25}$ we chose the ReNcell VM (RVM) hNSC, ${ }^{26}$ a more physiological relevant cell type, for further study of the neurogenesis. To confirm AICD-specific binding at the genomic locations of the 12 miRNA genes (Table 1), we performed independent ChIPPCR experiments in SH-SY5Y cells and hNSCs using another high specificity anti-AICD antibody (Supplementary Figure S1). These ChIP-PCR assays, using primer pairs for the 12 specified gene locations, confirmed that seven chromatin regions relevant to mir-3687, mir-3648, let-7a-1, mir-663, mir-3910, mir-193a and mir-595, were significantly enriched in both cell types (Figures $2 d$ and e). Thus, these data demonstrate that AICD interacts with the chromatin regions of mir-3648, mir-3687, let-7a-1, mir-663, mir-3910, mir-193a and mir-595.

AICD modulates the expression of miRNAs in hNSCs in a dual regulatory manner. AICD has been previously shown to contribute to the regulation of gene expression in a dual regulatory manner, both increasing and decreasing the expression of various genes. ${ }^{3}$ To reveal the biological significance of AICD-regulated miRNAs, we transduced AICD expression vector into hNSCs using a lentiviral vector and assessed the expression of the miRNAs using quantitative PCR (qPCR). After overexpression of AICD, the expression levels of miR-663 significantly increased, and those of both miR-3648 and miR-3687 were significantly decreased (Figure 2f). These results demonstrate that AICD may modulate the expression miRNAs in a dual regulatory manner.

As the binding sites for mir-663, mir-3648 and mir-3687 were situated near to the SSS of these miRNAs, the embedded regions were scanned using ChIP in both AICDoverexpressed and wild-type hNSCs. We found that the index of AICD-binding enrichment was significantly increased at regions of $-1150,-154$ and +375 bp away from the mir-663 SSS, but not at more distant regions or the control gapdh promoter region (Figure $2 \mathrm{~g}$ ). The mir-3648 and mir-3687 were clustered together in the genome, and the index of enrichment was significantly increased at -247 and +206 bp from the mir3648 SSS, but not at more distant regions or gapdh promoter region (Figure 2h). Similarly, ChIP-PCR validations were performed in wild-type hNSCs, and the result confirmed the 
a

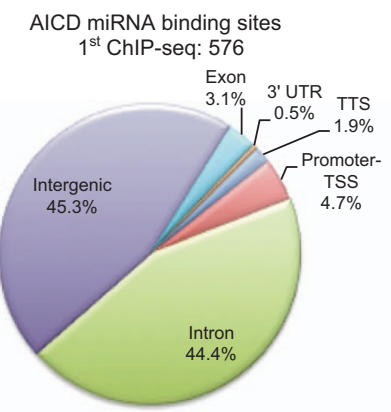

d

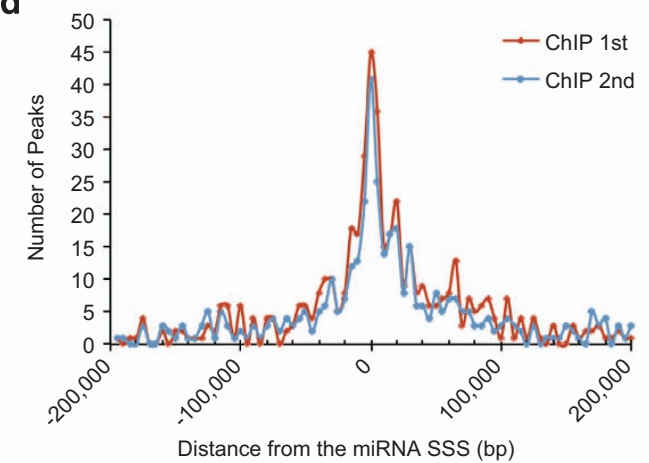

f Two ChIP-seq overlapped miRNAs: 207

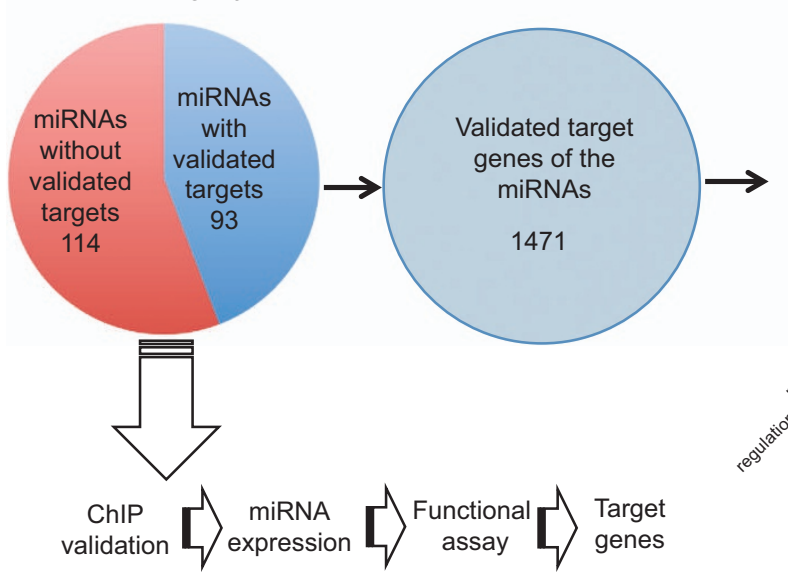

C

b
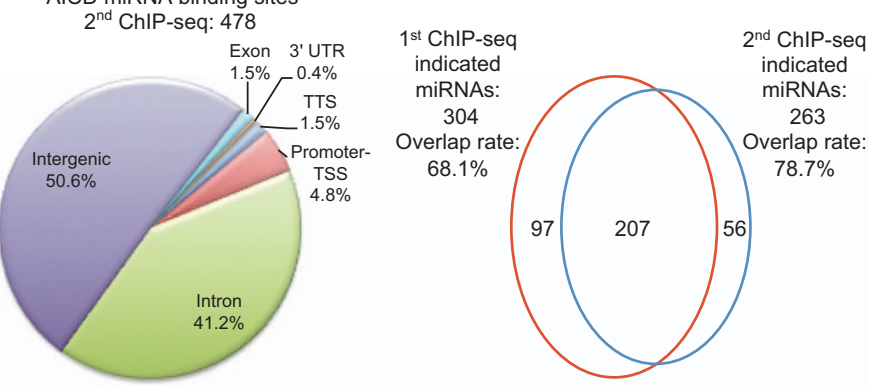

e

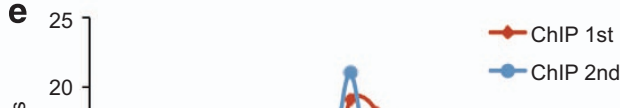

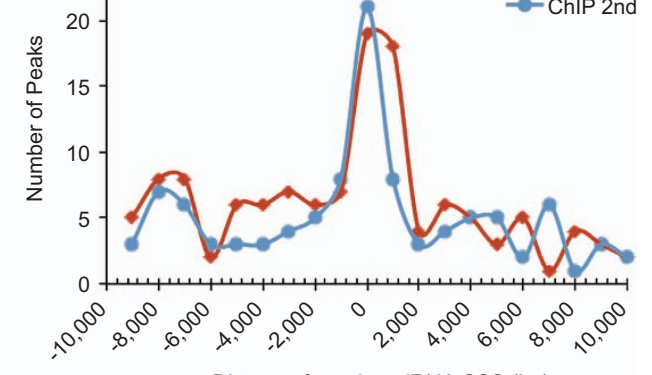

Distance from the miRNA SSS (bp)

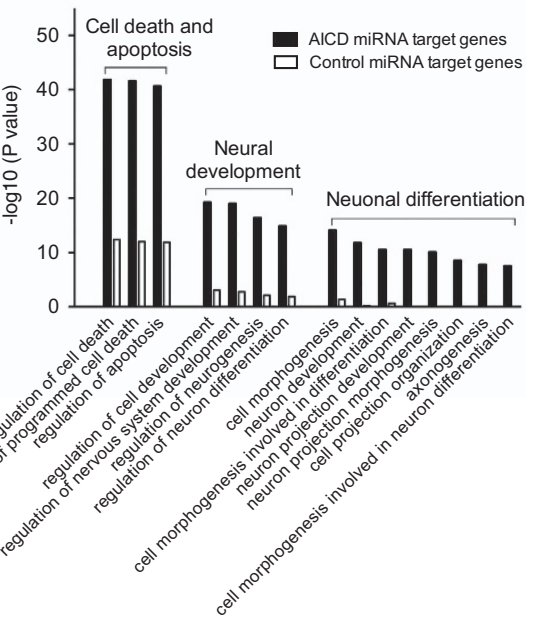

Figure 1 AICD is recruited to the miRNA-embedding regions in SH-SY5Y cells. (a and $\mathbf{b}$ ) Distribution of AICD miRNA-binding peaks from the first (a) and second (b) ChIPseq data sets in exons, introns, $3^{\prime}$-UTR, transcription terminal site (TTS), promoter-transcription start site (TSS) and intergenic regions of the genome. (c) Overlap of the miRNAs indicated by AICD-binding peaks from the first and second ChIP-seq data sets. (d and e) The distribution of the peaks within $200 \mathrm{~kb}$ (d) and $10 \mathrm{~kb}$ (e) of miRNA SSS. The $x$ axis shows the base distance from the miRNA SSS, noted as 0 . The $y$ axis is the number of peaks counted in the regions. (f) Functional annotation clustering analysis of the AICD ChIP miRNAs and schematic diagram of the strategy of this study

interactions between the endogenous AICD and these regions (Figures $2 \mathrm{i}$ and $\mathrm{j}$ ). Because the cis-acting elements are indicators of promoter activity, we questioned whether these two AICD-binding regions could be regulatory regions for these miRNAs. To this end, the distribution of Encyclopedia of DNA Elements (ENCODE) sites and CpG islands were retrieved from the UCSC Genome Browser. ${ }^{27}$ We found that the ENCODE sites and CpG islands were enriched in these regions. In addition, the distributions of ENCODE peaks were highly consistent with the distribution of our AICD ChIP enrichments in these regions (Supplementary Figure S2).
Therefore, these data indicate that AICD is recruited to the transcriptional regulatory regions of these miRNA genes.

AICD suppresses the neuronal differentiation of hNSCs. We have previously reported that AICD negatively regulates the neurogenesis of mouse NSC, ${ }^{15}$ a finding that was also supported by studies in the AICD transgenic mouse model. ${ }^{6}$ However, data from $A D$ patients and a number of mouse $A D$ models have revealed apparent conflicts with respect to whether neurogenesis is up- or downregulated. ${ }^{28}$ To investigate whether AICD restricts human neuronal differentiation, 

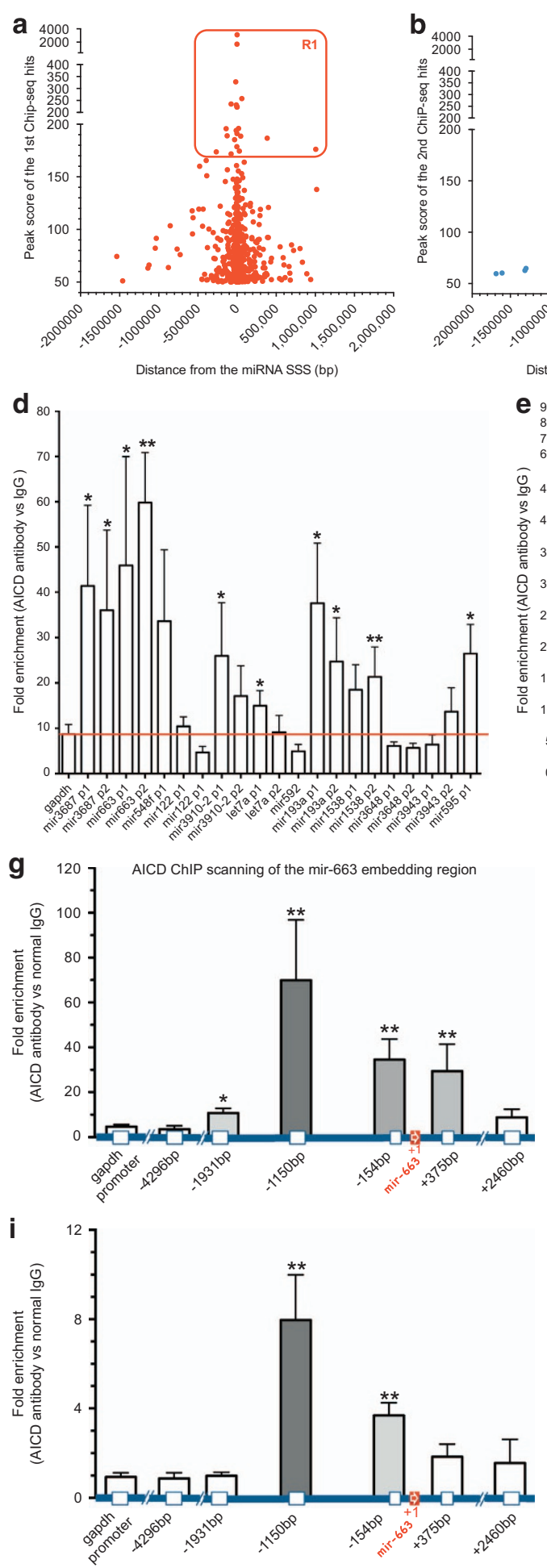

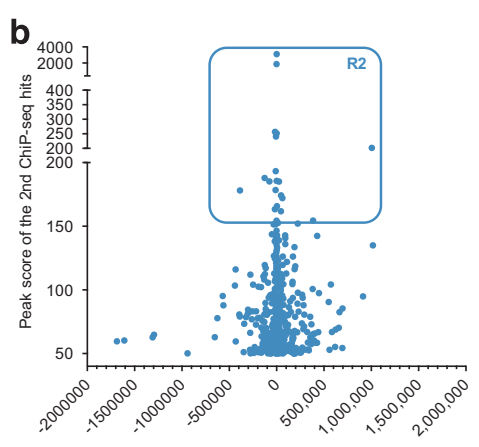

Distance from the miRNA SSS (bp)
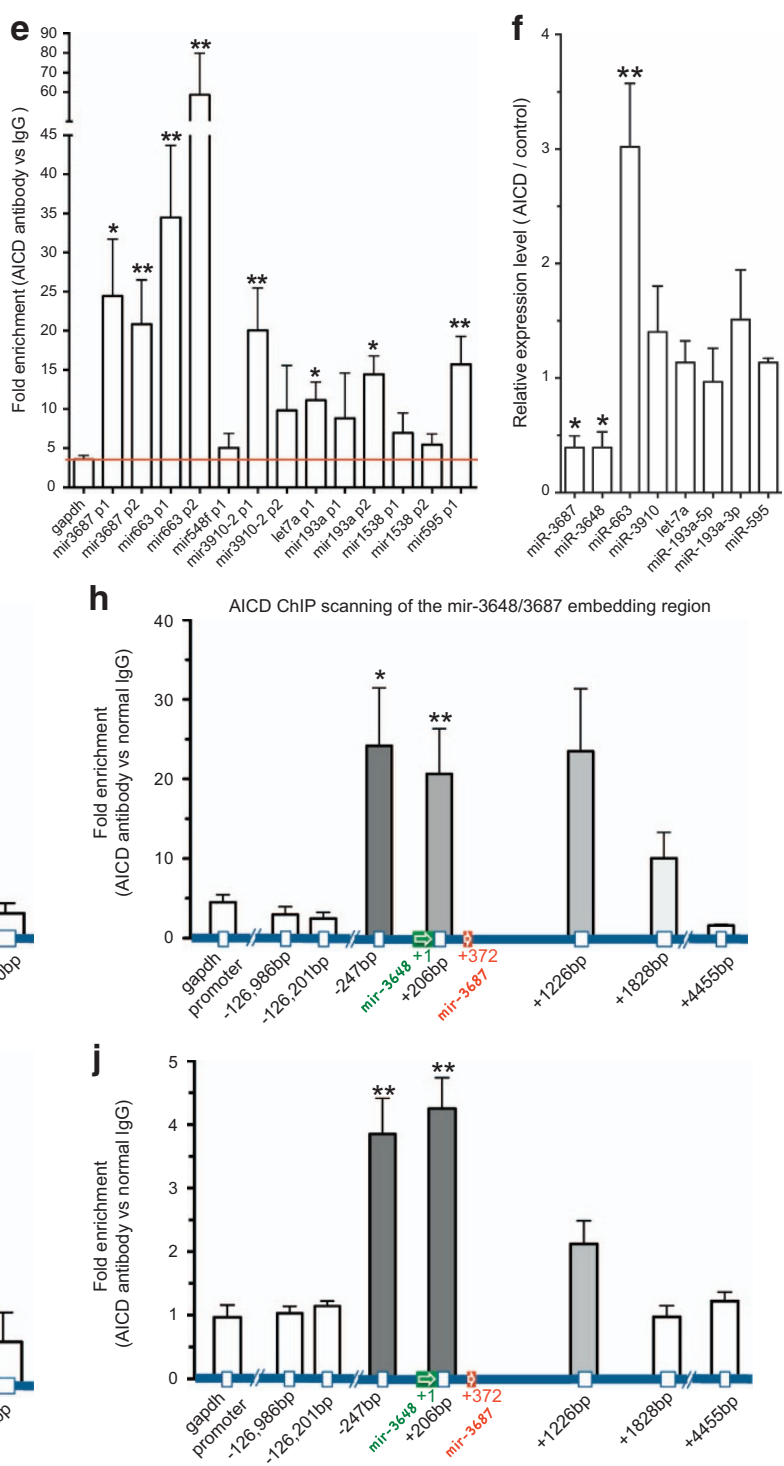

Figure 2 ChIP validation and miRNA expression assay for possible miRNAs regulated by AICD in hNSCs. (a and $\mathbf{b}$ ) Top 20 'peak score' miRNA hits were selected from the first (R1; a) and the second ChIP-seq data set (R2; b). (c) The overlapping 12 miRNA hits from R1 and R2 were selected for ChIP validation. (d) AICD ChIP validation performed in SH-SY5Y cells overexpressing AICD. The gapdh promoter region is set as control. (e) AICD ChIP validation performed in hNSCs overexpressing AICD. (f) Expression fold change of the eight miRNAs in hNSCs after AICD overexpression. (g) AICD ChIP scanning of the mir-663-embedding region in hNSCs overexpressing AICD. The gapdh promoter and other regions are set as control. The $x$ axis shows the base distance from the miRNA stem-loop start site, noted as +1 . (h) AICD ChIP scanning of the mir-3648- and mir-3687-embedding regions in hNSCs overexpressing AICD. (i and j) ChIP validation of the endogenous interaction of AICD with mir-663 (i), mir-3648 and mir-3687 (j) embedding regions in wild-type hNSCs. Quantification data were analyzed from at least three independent experiments (mean \pm S. E.M). ${ }^{*} P<0.05 ;{ }^{* *} P<0.01$ 
Table 1 Overlapped miRNA hits of the top 20 peaks from 2 ChIP-seq data sets

\begin{tabular}{|c|c|c|c|c|c|c|}
\hline Description & Chr & Start & End & Peak score & Annotation & Distance to SSS (bp) \\
\hline mir-3687 & chr21 & 9825273 & 9827785 & 3100 & TTS & 321 \\
\hline mir-663 & chr20 & 26188340 & 26191020 & 1783.49 & Promoter-TSS & -733 \\
\hline mir-548f-5 & chr13 & 36531932 & 36532876 & 292.21 & Intron & -17000 \\
\hline mir-122 & chr18 & 56179601 & 56180702 & 215.02 & Intron & 61798 \\
\hline let-7a-1 & chr9 & 96927827 & 96929643 & 212.295 & Intergenic & -9435 \\
\hline mir-3910-2 & chr9 & 94472980 & 94474733 & 210.17 & Intergenic & -75228 \\
\hline mir-592 & chr7 & 125691975 & 125692956 & 188.995 & Intergenic & 1005759 \\
\hline $\operatorname{mir}-3648^{a}$ & chr21 & 9698762 & 9700581 & 188.45 & Intergenic & -126353 \\
\hline mir-193a & chr17 & 29876126 & 29877625 & 186.215 & Intergenic & -10141 \\
\hline mir-3943 & chr7 & 43236886 & 43238815 & 181.425 & Intron & 47413 \\
\hline mir-1538 & chr16 & 69599285 & 69600694 & 172.74 & Promoter-TSS & -136 \\
\hline mir-595 & chr7 & 157939829 & 157940996 & 170.62 & Intron & 385055 \\
\hline
\end{tabular}

${ }^{a}$ mir-3687 and mir-3648 are clustered in the genome, and two different binding peaks are found for them

we overexpressed AICD in hNSCs, and we found that the number of neuronal-like cells in the AICD-transfected group was significantly decreased in comparison with the control group as demonstrated by expression of the neuronal marker $\beta$ III-tubulin (Figures $3 a$ and b).

miR-663 suppresses neuronal differentiation of hNSCs. To determine which of the AICD-regulated miRNAs are involved in inhibiting neuronal differentiation, we transfected molecular mimics of miR-663, miR-3687 or miR-3648 into hNSCs. After differentiation, the transfected hNSCs were assessed for neuronal differentiation by flow cytometry and immunohistochemistry, using antibodies against $\beta$ III-tubulin. Flow cytometry analysis showed that the number of $\beta$ IIItubulin-positive cells was significantly decreased in cells overexpressing miR-663 (Figures $3 c$ and d), but not in those overexpressing miR-3648 or miR-3687 (Supplementary Figures $\mathrm{S} 3 \mathrm{a}$ and $\mathrm{b}$ ). Similar results were obtained by quantitative analyses of the immunohistochemistry (Figures $3 e$ and $f$, Supplementary Figures $S 3 c$ and $d$ ). We also estimated the percentage of $\beta$ III-tubulin-positive cells after transfection of the corresponding antisense inhibitors and the percentage of GFAP (an astroglial marker)-positive cells after transfection with the miRNAs; however, no significant changes were detected (Supplementary Figures S3e-h). These results demonstrate that, among the AICD-regulated miRNAs, miR-663 mediates suppression of neuronal differentiation in hNSCs.

To further confirm that AICD inhibits neuronal differentiation through miR-663, we investigated whether inhibition of miR-663 could rescue AICD-mediated suppression of neuronal differentiation. hNSCs were co-transfected with miR-663 antisense inhibitor and AICD. After differentiation, both flow cytometry and immunohistochemistry showed that the number of $\beta$ III-tubulin-positive cells was significantly increased after co-transfection of miR-663 antisense inhibitor with AICD (Figures $3 g-j$ ). These observations indicate that miR-663 is a mediator for the AICD suppression of neuronal differentiation in hNSCs.

miR-663 targets multiple genes in hNSCs. Vertebrate miRNAs have been reported to target multiple genes at the transcriptional level through interaction with untranslated regions (UTRs) to perform their biological function. ${ }^{29-31}$ Interestingly, miR-663, a member of primate-specific miRNA family, has recently been found to be associated with cancer, through the regulation of different target genes in cancer cells. $^{32,33}$ To identify the genes targeted by miR-663 in hNSCs, the miR-663 mimics were transfected into hNSCs, and mRNA was purified and profiled on gene expression microarrays (Supplementary Figure S4a). We found that 123 and 253 genes were significantly downregulated $(P<0.001)$ at 12 and $24 \mathrm{~h}$ after miR-633 transfection, respectively. In addition, 91 genes were significantly downregulated at both 12 and $24 \mathrm{~h}$ (Figure $4 \mathrm{a}$ and Supplementary Table S6).

In order to predict and identify the possible target genes of miR-663 among the genes with differential expression, we checked whether any are predicted targets using Targetscan. ${ }^{29}$ We found that the predicted direct targets of miR-663 were enriched in the downregulated genes as compared with the total genes in the study, and greatly enriched in the 91 downregulated genes (Figure 4b). Furthermore, we observed significantly over-representation of gene inhibition among the Targetscan predicted genes as compared with all the other downregulated genes (Figure 4c). ${ }^{34}$ To evaluate the microarray-downregulated genes solely based on the sequence motifs of their $3^{\prime}$-UTRs, the motif discovery tool MEME was used to computationally search the $3^{\prime}$-UTR sequences of the downregulated genes. ${ }^{35}$ We found a highly significant consensus sequence 'CCCCGCCCC' in the $3^{\prime}$-UTR sequences of the downregulated transcripts. This consensus motif was complementary to the positions 2-8 from the $5^{\prime}$ end of miR-663, the crucial seed region required for target recognition (Figure 4d). ${ }^{31}$ Moreover, DIANA-mirExTra hexamer analysis confirmed that the direct targets of miR-663 were enriched in the 91 downregulated genes (Figure $4 \mathrm{e}){ }^{36}$

AICD/miR-663-downregulated genes inhibit neuronal differentiation. To identify downstream genes of AICD/ miR-663 signaling during neurogenesis, we performed gene expression microarray analysis in AICD-overexpressing hNSC cells. In brief, hNSCs were transduced by AICD, and EGF and bFGF were withdrawn to stimulate cell differentiation at $48 \mathrm{~h}$ post transduction, when miR-663 was upregulated (Figure 2f). After another $24 \mathrm{~h}$, total RNA was prepared for microarray analysis. We found that 853 genes were 
a

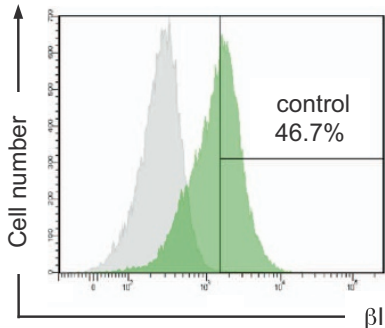

C
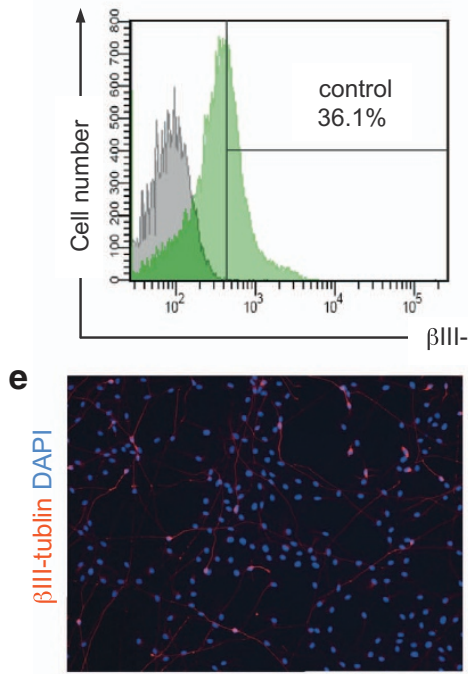

control

9

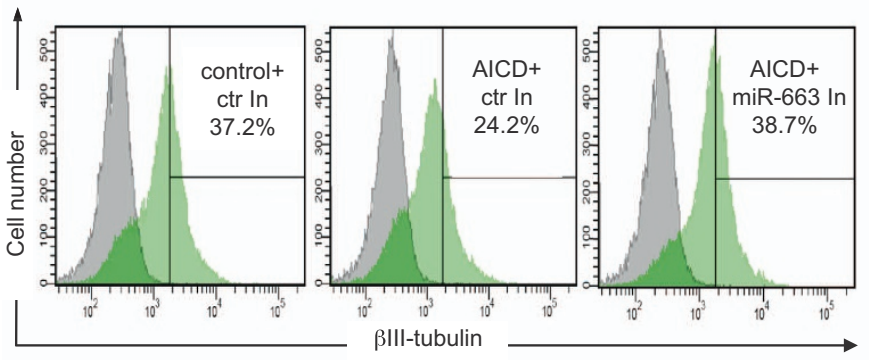

i

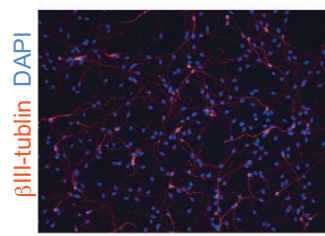

control + ctr In
Blll-tubulin
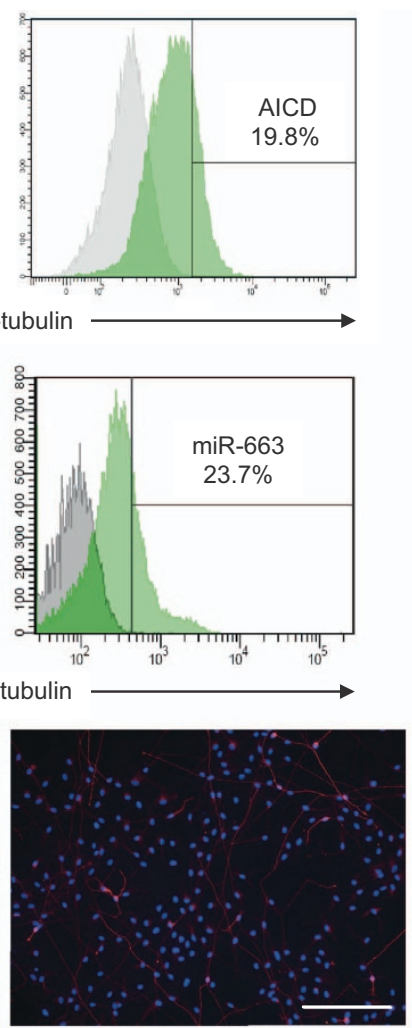

miR-663

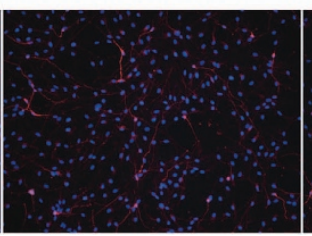

AICD + ctr In

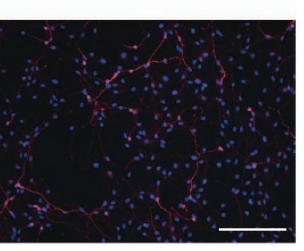

AICD + miR-663 Inhibitor
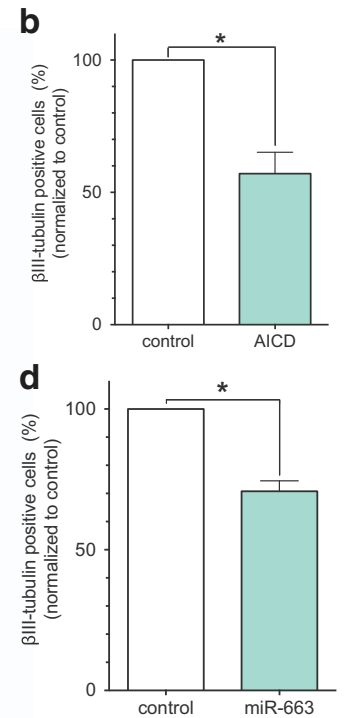

f
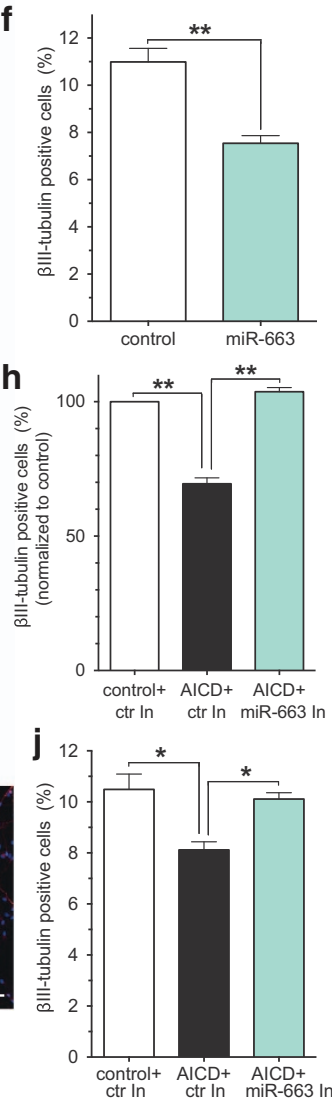

Figure 3 AICD inhibits hNSC differentiation through miR-663. (a and $\mathbf{b})$ Representative histograms (a) and quantification (b) of $\beta$ III-tubulin detection using flow cytometry in differentiated hNSCs transfected with AICD and control (green: $\beta$ III-tubulin; gray: isotype control). (c and d) Representative histograms (c) and quantification (d) of $\beta$ III-tubulin detection using flow cytometry in differentiated hNSCs transfected with miR-663 mimics and control (green: $\beta$ IIl-tubulin; gray: isotype control). (e and f) Representative images (e) and quantification (f) of $\beta$ III-tubulin detection using immunohistochemistry in differentiated hNSCs transfected with miR-663 mimics and control. Scale bar $=200 \mu \mathrm{m}$. (g and $\mathbf{h})$ Representative histograms $(\mathbf{g})$ and quantification $(\mathbf{h})$ of $\beta$ III-tubulin detection using flow cytometry in differentiated hNSCs transfected with control vector plus non-targeting control inhibitors (ctr In), AICD plus non-targeting control inhibitors, AICD plus miR-663 antisense inhibitors, respectively (green: $\beta$ III-tubulin; gray: isotype control). (i and j) Representative images (i) and quantification (j) of $\beta$ III-tubulin detection by immunohistochemistry in differentiated hNSCs transfected with control vector plus non-targeting control inhibitors (ctr In), AICD plus non-targeting control inhibitors, AICD plus miR-663 antisense inhibitors, respectively. Scale bar $=200 \mu \mathrm{m}$. Quantification data were analyzed from at least three independent experiments (mean \pm S.E.M). ${ }^{*} P<0.05 ;{ }^{*} P<0.01$ 
a

miR-663-12h down-regulated genes

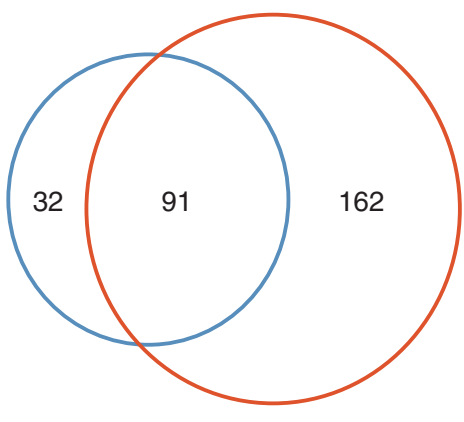

b Percentage of genes with the miR-663 targeting sites

$12 \mathrm{~h}$

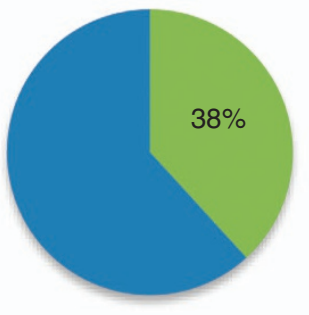

C

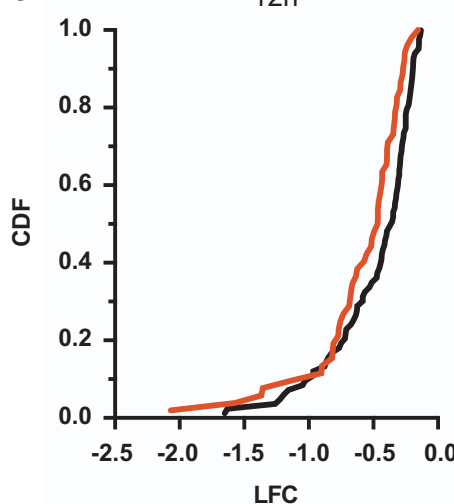

d

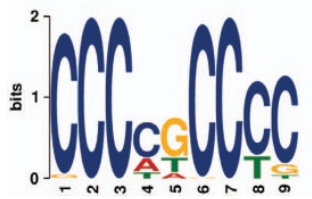

UTRs with consensus: $44.21 \%$

E-value: $6.9 \times 10^{-047}$

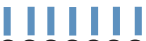

CCCCGCCCC

$24 \mathrm{~h}$
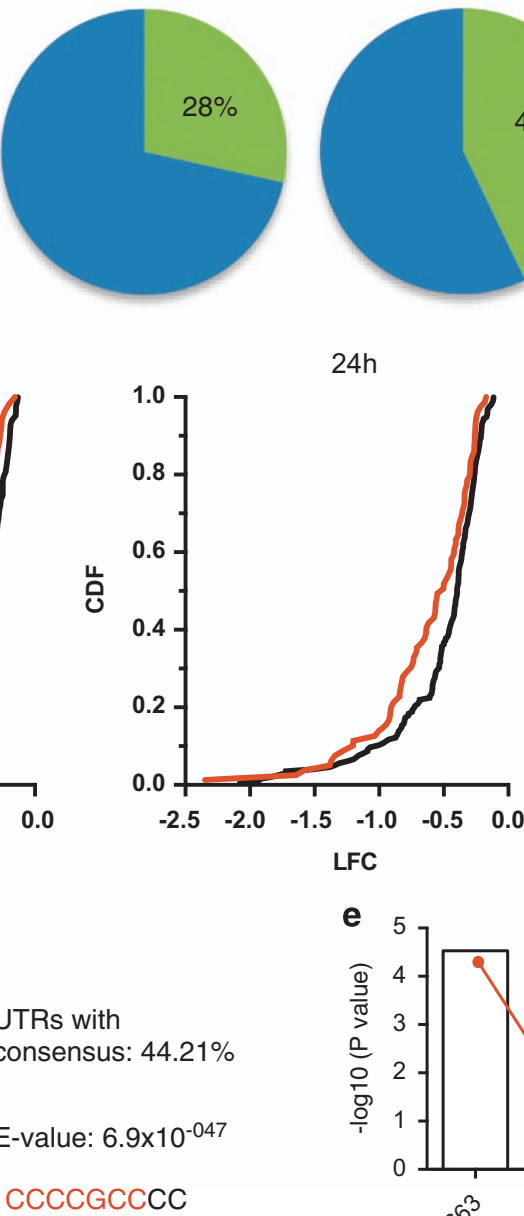

LFC
miR-663-24h

down-regulated

genes

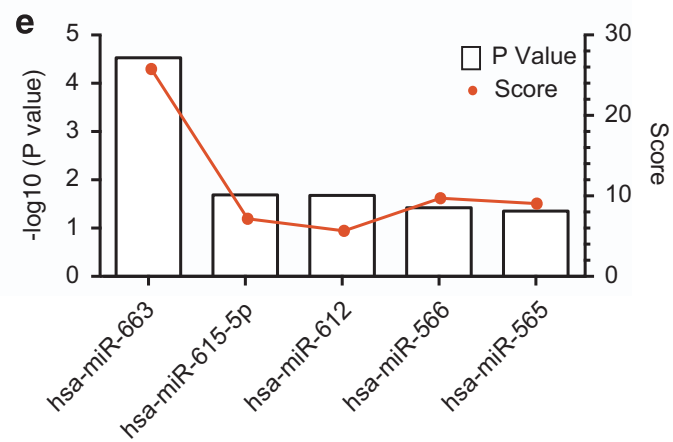

Figure 4 Multiple genes are downregulated after miR-663 mimics transfection in hNSCs. (a) Ninety-one genes are downregulated significantly downregulated $(P<0.001)$ at both 12 and $24 \mathrm{~h}$ after miR-663 transfection. (b) Percentage of the genes containing miR-663 target sites predicted by Targetscan in the miR-663 microarray downregulated genes and total genes. (c) Cumulative distribution plots of log2-transformed gene expression fold changes (LFCs) for downregulated genes containing miR-663 target sites predicted by TargetScan (red) and all other downregulated genes (gray) after miR-663 transfection. The $y$ axis shows cumulative distribution function (CDF) of LFC distribution. ( $P$-values $<0.05$ by Wilcoxon test). (d) Representative motif found by MEME in the $3^{\prime}$-UTRs of the genes downregulated at both 12 and $24 \mathrm{~h}$. The sequence logo of the weight matrix was constructed by MEME. (e) DIANA-mirExTra algorithm shows miR-663 hemxamers are enriched in the $3^{\prime}$-UTRs of the genes downregulated at both 12 and $24 \mathrm{~h}$. The $P$-values and scores of the top five most significant miRNAs calculated with DIANA-microT are shown 
significantly downregulated $(P<0.05)$ after AICD overexpression (Supplementary Figure S4 and Table S7). Subsequently, we intersected the miR-663-downregulated gene profiles $(P<0.001)$ with the AICD-downregulated gene expression profile, and revealed that seven genes were downregulated by both AICD and miR-663 (Figure 5a). In addition, we overlapped the miR-663-downregulated gene profiles $(P<0.05$; Supplementary Figure S4 and Table S8) with both the AICD-downregulated gene expression profile and the Targetscan-predicted genes of miR-663, and revealed that another 11 genes were downregulated by both AICD and miR-663 (Figure 5b). In total, we found 13 genes for further validation (Figure 5c). After qPCR validation, eight genes were selected for further flow cytometry analysis of neuronal differentiation, and we found that siRNA knockdown of PTP4A1, FBXL18, CDK6 and ZFAND3 resulted in significant suppression of neuronal differentiation (Figure $5 \mathrm{~d}$ ). In addition, the overall effect of knockdown of these genes was the inhibition of neurogenesis in the hNSCs (Figure 5e). To further determine which genes are the direct targets of miR-633, wild-type and mutant UTR segments possessing the seed matches from these four genes were cloned into dual-luciferase vectors. When co-transfected with the miR-663, repression of the reporter gene was observed in the $3^{\prime}$-UTR of the FBXL18 and $5^{\prime}$-UTR of the CDK6 in comparison with the corresponding mutant UTRs (Figure 5f). The reporter assay result demonstrates that AICD/miR-663 can downregulate the expression of $F B X L 18$ and $C D K 6$ directly.

\section{Discussion}

In the present study, using genome-wide ChIP-seq we have identified a set of miRNAs regulated by AICD. Furthermore, we have shown that AICD acts as a transcriptional regulator to modulate the expression of miRNAs. Importantly, we have demonstrated that miR-663 has an important role in suppressing neuronal differentiation of hNSCs in vitro and have identified an AICD downstream mechanism suppressing neurogenesis in hNSCs (Figure 6).

APP is considered having a key role in the pathogenesis of $A D$, as cleavage of APP produce $A \beta$ peptide that is deposited in the brain of $A D$ patients. In addition to $A \beta$, the ectodomain of APP has been reported to have neuroprotective effects and mediate the physiological functions of APP. ${ }^{16}$ The intracellular domain of APP is also believed to be functionally important, as numerous proteins interact with this region to regulate the processing and function of APP.,37 However, there is controversy over the transcriptional regulation of AICD. AICD has been first implicated with transcriptional activity through the Gal4 reporter assay. ${ }^{17}$ Following studies have demonstrated that AICD regulates the transcription of genes,

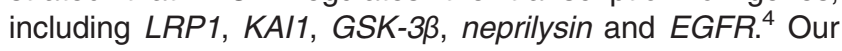
results, we believe, resolved some of these discrepancies by providing clues that AICD interacts with the chromatin directly to affect transcription in both of the overexpression and endogenous systems; nevertheless, the signal transduction model of AICD and its relation with the binding proteins need to be further explored. In addition, the APP family proteins, APLP1 and APLP2, also have similar C terminus as APP. ${ }^{38}$ $A$ recent study has shown the nuclear signaling function for
APP, and APLP2 is absent in APLP1. ${ }^{39}$ However, the specific interactions of these proteins with chromatin, and the differences of nuclear function among the APP family members, need to be determined in the future.

As miRNAs are important regulators of gene expression, we focus on the possibility that AICD modulates the expression of miRNAs. In search for the miRNAs regulated by AICD, we performed ChIP-seq experiments in APP695-overexpressing cells, given that AICD turns over rapidly, whereas it is preferentially produced from the APP695 isoform. ${ }^{40}$ It is established that miRNAs are first transcribed as primary miRNAs (pri-miRNAs). ${ }^{21,22}$ As the pri-miRNAs vary in length from a few hundred bases up to hundreds of kilobases, the regulatory regions for the miRNAs could be located up to several megabases from the miRNA stem-loop sequence in the genome. ${ }^{41,42}$ Consequently, a wider distance criterion ranging within 2 megabases from the SSS was used to associate the ChIP-seq peaks with miRNAs. Using our ChIPseq data, we discovered a set of AICD-regulated miRNAs, and the subsequent FAC analysis suggested a promising potential that AICD may regulate apoptosis and neurogenesis through miRNAs. However, the wider distance criterion, together with the overexpression system, could result in nonspecific binding sites in the ChIP-seq data. For this reason, both ChIP validation of endogenous binding and functional validation are required in the specific cells before drawing any conclusions.

In vivo neuronal development is a well-orchestrated process containing several specific cell stages, such as immature precursor, neuronally committed precursor, immature neuron and mature neuron. ${ }^{43} \mathrm{~A}$ number of miRNAs have also been demonstrated to regulate neural stem cell proliferation, differentiation and maturation during the neuronal development. ${ }^{44}$ miR-663, expressed only in Homo sapiens, Macaca mulatta and Pantroglodytes, belongs to the primatespecific miRNAs. ${ }^{45}$ The function of miR-663 has been reported to be mainly associated with cancer biology. In acute myeloid leukemia patients, the miR-663 promoter is hypermethylated and influences leukemia cell differentiation. ${ }^{46,47}$ In addition, miR-663 targets the expression of multiple genes, such as TGF $\beta 1, p 21, H$-ras, eEF1 and HSPG2, to regulate cancer cell proliferation and chemotherapy resistance. ${ }^{32,33,48-50}$ Moreover, miR-663 is related to smooth muscle cell phenotypic switch in the cardiovascular system. ${ }^{51}$ Briefly, these previous studies provide a clue that miR-663 is able to influence cell proliferation and differentiation; however, its role in the nervous system is not well understood. Our findings show that miR-663 can decrease the number of $\beta$ IIItubulin-positive cells during neuronal differentiation. Given that miR-663 might influence the proliferation of hNSCs, we performed a BrdU incorporation assay as well; however, no significant differences were found (Supplementary Figure S5a and b). We also evaluated the morphology of differentiated neurons after miR-663 overexpression by measuring the length of neurites. Preliminary result showed that miR-663 did not decreased the length significantly (Supplementary Figure S5c). Therefore, the exact role of miR-663 at different stages of the neuronal differentiation needs to be explored in future studies. 

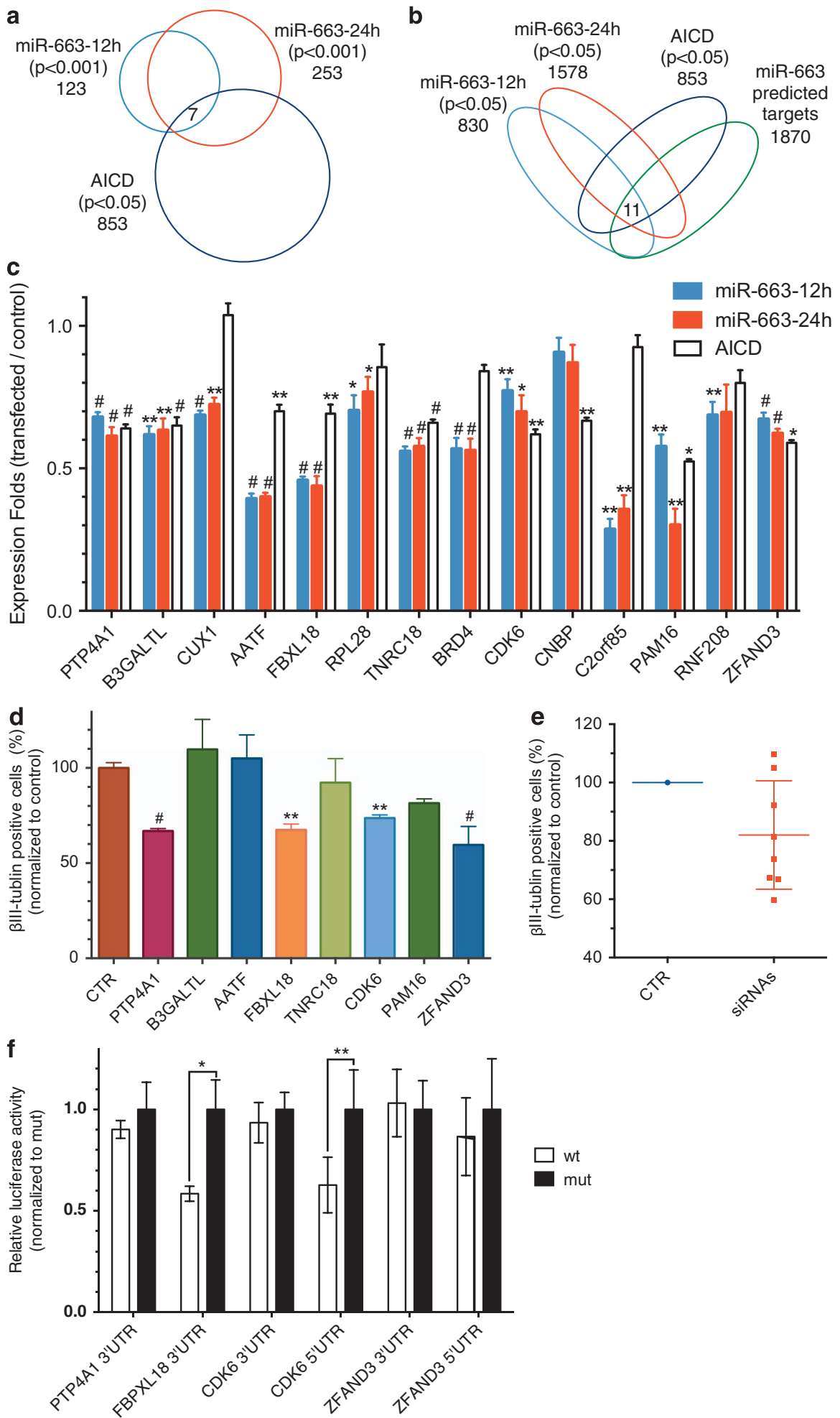

Figure 5 Genes downregulated by both miR-663 and AICD are associated with neurogenesis. (a) Three-way Venn diagram demonstrating the overlap among the miR-663 $(P<0.001)$ and AICD $(P<0.05)$ microarray-downregulated genes. (b) Four-way Venn diagram demonstrating the overlap among the miR-663 $(P<0.05)$, AICD $(P<0.05)$ microarray-downregulated genes and genes with miR-663 target sites predicted by Targetscan. (c) Validation of the gene expression fold change using qPCR. (d) Quantitative flow cytometry analysis of $\beta$ III-tubulin expression in differentiated hNSCs transfected with siRNAs and non-targeting control. (e) Overall effects of the downregulation of these genes by siRNA on neuronal differentiation. (f) Dual-luciferase reporter assay shows that miR-663 directly represses the expression luciferase genes bearing $5^{\prime}$-UTR segments of CDK6 and $3^{\prime}$-UTR segments of FBPXL18. Quantification data were analyzed from at least three independent experiments (mean \pm S.E.M). ${ }^{*} P<0.05$, ${ }^{\star \star} P<0.01$, ${ }^{\#} P<0.001$ 


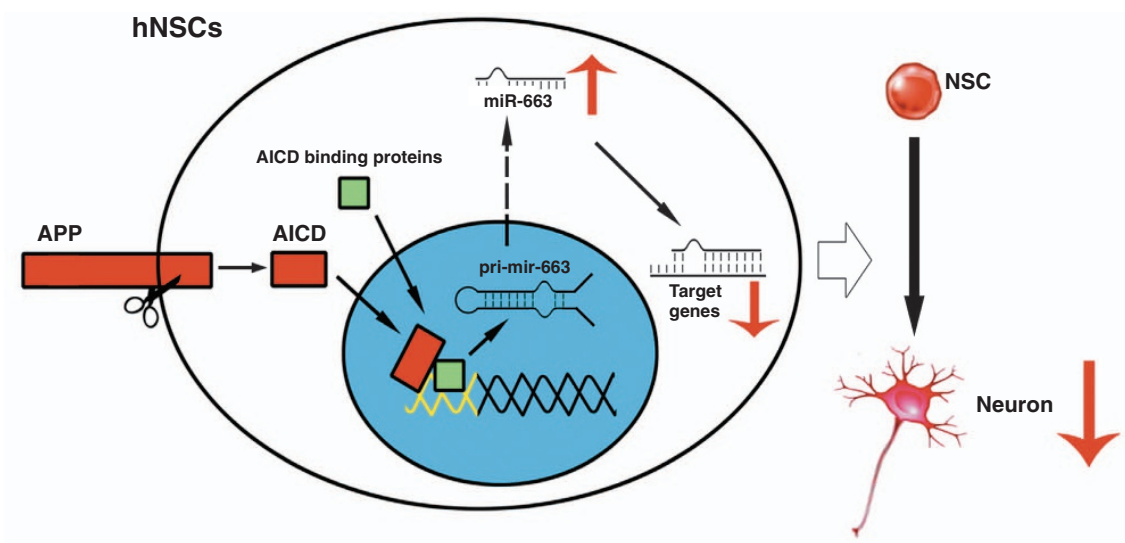

Figure 6 Proposed model of the APP-AICD-miR-663 signaling in hNSC differentiation. APP is located at the plasma membrane of hNSCs. Ligand binding to APP triggers an intramembrane cleavage of APP to generate the AICD, which translocates to the nucleus. Nuclear AICD promotes the expression of miRNAs, such as miR-663, which suppress the expression of genes such as FBXL18 and CDK6, to inhibit hNSC neuronal differentiation

As multiple different miR-663 target genes have been reported, we hypothesized that miR-663 also targets multiple genes to perform its function in the nervous system. Using gene expression microarrays, we found that multiple genes with miR-663 predicted that target sites were downregulated in hNSCs. After validation, we identified two direct target genes, FBXL18 and CDK6, which could contribute to the AICD/miR663 inhibition of neurogenesis. FBXL18 is a member of F-box protein family, which could form the SKP1CUL1-F-box protein (SCF) complex to act as a protein ubiquitin ligase ${ }^{52}$ and some of the SCF complexes have already been suggested to control neuronal differentiation, ${ }^{53,54}$ yet the exact role of $F B X L 18$ remains to be demonstrated. The other gene, $C D K 6$, is essential for the expansion of neuronally committed precursors and the production of newborn neurons. ${ }^{43}$ In addition, CDK6 is a mediator of PAX6's modulation of cortical progenitor cell proliferation. ${ }^{55}$ Therefore, it is possible that miR-663 inhibits neuronal differentiation through CDK6.

The current study demonstrates that the abnormal processing of APP might lead to aberrant AICD regulation of miRNA expression, which may be linked to abnormal intracellular signaling. However, to address the relevance of miRNAs with $A D$, further studies performed in vivo or in human tissue are required. It is also important to acknowledge that only a small fraction of miRNA candidates were selected according to the peak-calling score and distance in this study. Interestingly, the recently identified miR-574-5p, which is associated with the neuronal inhibition by APP, ${ }^{19}$ is also included in both of the two data sets of our ChIP-seq miRNAs (Supplementary Tables S1 and S2). However, the direct binding of AICD to the genomic region surrounding miR-574 remains to be determined. Thus, for the remaining miRNAs, their regulation by AICD and biological roles need to be further defined in specific cell types and biological assays. Considering the recent concern on the confidence of the miRNA in the miRBase, the miRNA candidates could also be filtered according to the confidence of the miRNA in the future. ${ }^{56,57}$

In conclusion, the present study has identified the role of AICD-regulated miR-663 in hNSC differentiation and provided one molecular mechanistic insight into AICD signaling in human neurogenesis in vitro. This may have ramifications in the context of the physiological role of APP, as well as more broadly in $A D$ research. Further understanding of these mechanisms might shed light on the cellular processes of neurodegenerative diseases and may offer an opportunity for pharmacological intervention.

\section{Materials and Methods}

ChIP-Seq. ChIP-seq was performed as previously described. ${ }^{58} \mathrm{~A}$ SH-SY5Y cell line overexpressing APP was generated by the stable transfection of a pcDNA4 construct carrying APP. Nuclear lysates were cross-linked with $1.5 \mathrm{mM}$ dithiobis succinimidyl propionate (DSP) and $1 \%$ formaldehyde. Chromatin for the immunoprecipitation was sheared to fragments below $500 \mathrm{bp}$. Quality-control checks on the DNA were run using the Agilent Bioanalyzer 2100 (Mulgrave, VIC, Australia). Antibody against C-terminal APP (BR188 from M. Goedert, University of Cambridge, UK) ${ }^{25}$ and control rabbit IgG were used. Immunoprecipitated DNA from ChIP experiments was subjected to deep DNA sequencing. The reads were aligned against the hg19 Human Genome Assembly (GRCh37, February 2009) and peak calling was performed using the MACS (Model-based Analysis for ChIP-Seq) software. ${ }^{59}$ Significant binding sites within 2 megabases from the miRNA SSS were selected as potential candidates.

Cell culture and transfection. The SH-SY5Y cell line was grown in Dulbecco's modified Eagle's medium (DMEM; Gibco, Mulgrave, VIC, Australia), supplemented with $10 \%$ heat-inactivated fetal bovine serum (Gibco), $1 \%$ penicillin/ streptomycin (Gibco). The RVM hNSC line (Millipore, Bayswater, VIC, Australia) was cultured and differentiated according to the published protocols. ${ }^{26}$ For proliferation, RVM cells were cultured in the RVM medium (DMEM/F12 medium (Gibco) with B27 (Gibco), $10 \mathrm{U} / \mathrm{ml}$ heparin (Sigma-aldrich, St. Louis, MO, USA) and 1\% Gentamicin (Gibco)) with $20 \mathrm{ng} / \mathrm{ml}$ EGF (Peprotech, Rocky Hill, NJ, USA) and $10 \mathrm{ng} / \mathrm{ml} \mathrm{bFGF}$ (Peprotech). For differentiation, the cells were seeded on laminin (Invitrogen, Mulgrave, VIC, Australia)-coated plates, the EGF and bFGF were removed, and $1 \mathrm{mM}$ dibutyrl-cAMP (Calbiochem, Lafayette, CO, USA) and $2 \mathrm{ng} / \mathrm{ml}$ GDNF (Peprotech) were added to the differentiation media. The miRNA mimics and heparin inhibitors (Dharmacon, Lafayette, CO, USA) as well as the siRNAs (Silencer Select validated and pre-designed siRNA, Ambion, Mulgrave, VIC, Australia) were transfected into the cells using the Amaxa Nucleofector Kit for mNSC (LONZA, Mount Waverley, VIC, Australia) according to the manufacturer's manual. The knockdown efficiency of the siRNAs was validated by qPCR. The siRNA sequences are listed in Supplementary Table S9.

Chromatin immunoprecipitation. ChIP assays were carried out as described by Sandoval et al. ${ }^{60}$ Cells were fixed with DSP (Thermo Scientific, Waltham, MA, USA) for $45 \mathrm{~min}$ before fixing with $1 \%$ formaldehyde for $10 \mathrm{~min}$. Glycine $(0.2 \mathrm{mM})$ was used to quench the reaction. After cell lysis, chromatin extracts were sonicated (Bioruptor, Denville, NJ, USA) to fragments of $\sim 200-500 \mathrm{bp}$ and pre-cleared by Dynabeads Protein A (Invitrogen). The pre-cleared chromatin extract was incubated with C-terminal APP antibody (Invitrogen, cat no. 512700) overnight at $4^{\circ} \mathrm{C}$, and then it was incubated with Dynabeads Protein $\mathrm{A}$ for $4 \mathrm{~h}$ at 
$4^{\circ} \mathrm{C}$. The beads were then washed, and the chromatin-protein-antibody complexes were eluted. After treatment with Proteinase K (Finnzymes, Waltham, MA, USA), the eluted DNA was purified by the PCR purification kit (Qiagen, Chadstone, VIC, Australia). QPCR was performed to determine the fold enrichment of immunoprecipitated DNA. qPCR reaction mixes were assembled using the SYBR Green master mix (Roche, Indianapolis, IN, USA). The reactions were performed on the LightCycler 480 System (Roche) using following cycling parameters: $95^{\circ} \mathrm{C}$ for $10 \mathrm{~min}$, and then 45 cycles of $95^{\circ} \mathrm{C}(10 \mathrm{~s}), 60^{\circ} \mathrm{C}(10 \mathrm{~s})$ and $72{ }^{\circ} \mathrm{C}(10 \mathrm{~s})$ followed by a melting curve analysis. All reactions were performed with three technical replicates. Fold change values were normalized against input DNA and compared with the rabbit lgG control. The gapdh promoter region was selected as the negative control. The primer sequences for qPCR are listed in the Supplementary Table S9.

Functional annotation clustering analysis. The validated target genes of the 207 miRNAs were retrieved from the miRWalk (http://www.umm.uni-heidelberg. de/apps/zmf/mirwalk/mirnatargetpub.html), and 1471 validated genes, which were targeted by 93 of miRNAs, were found. The 93 control miRNAs, which were supposed to be not influenced by AICD, were randomly selected from the 1522 miRNAs with validated target genes, and these miRNAs were not included in the two ChIP-seq data sets. The random selection was performed by the Excel (Microsoft, Redmond, WA USA). The validated target genes of control miRNAs were also retrieved from miRWalk. The Functional annotation analysis was performed in the David Functional Annotation Clustering Analysis website (http://david.abcc.ncifcrf.gov/home.jsp), using the default setting of the medium classification stringency. The P-values (with Benjamini correction) of GO terms were compared.

Lentivirus construction, production and transduction. The secondgeneration, self-inactivating bicistronic lentiviral transfer vector, pWPI (kindly provided by D. Trono), was used to produce lentivirus for transfection. The open reading frames for human AICD59 were cloned upstream of an IRES-eGFP cassette by blunt-end ligation to generate the vectors pWPI-AICD-IRES-eGFP. Vira stocks were generated as previously described by Siatskas et al. ${ }^{61}$ When the cells had grown to $70 \%$ confluency, pWPI-AICD-IRES-eGFP lentiviral stock was added to the cell culture medium with $4 \mu \mathrm{g} / \mathrm{ml}$ protamine sulfate. The cells were incubated with lentivirus overnight before changing the medium.

Statistical analysis. Quantitative data are expressed as mean \pm S.E.M. Statistical significance between any two groups was determined by the two-tailed Student's $t$-test or one-way ANOVA analysis using the GraphPad Prism5 (GraphPad software, La Jolla, CA, USA). $P$-values less than 0.05 were considered significant.

\section{Conflict of Interest}

The authors declare no conflict of interest.

Acknowledgements. This work was supported by grants from the Professorial Fellowship of Monash University, Australia and the Talent Program of Yunnan Province, China to ZCX; National Basic Research Program of China (973 program; 2013CB945602) and the National Natural Science Foundation of China (31171313 and 81271424), to QHM; the Key Project of Chinese Ministry of Education (NO211170), China, to SY; from the Agency for Science, Technology and Research (A*STAR) Singapore Stem Cell Consortium (SSCC/08/016) to GSD, QHM and ZCX; the National Health and Medical Research Council of Australia (APP1053621) and the Eva and Les Erdi AUSiMED Fellowship in Neurological Diseases to CCAB; the McDonald Fellowship of Multiple Sclerosis International Federation to RS. We thank Drs Michel Goedert and Graham Fraser (Cambridge, UK) for providing the AICD BR188 antibody; Dr. Didier Trono (EPFL, Switzerland) for the lentiviral vectors.

1. Selkoe DJ. Cell biology of protein misfolding: the examples of Alzheimer's and Parkinson's diseases. Nat Cell Biol 2004; 6: 1054-1061.

2. LaFerla FM, Green KN, Oddo S. Intracellular amyloid-beta in Alzheimer's disease. Nat Rev Neurosci 2007; 8: 499-509.

3. Muller T, Meyer HE, Egensperger R, Marcus K. The amyloid precursor protein intracellula domain (AICD) as modulator of gene expression, apoptosis, and cytoskeletal dynamicsrelevance for Alzheimer's disease. Prog Neurobiol 2008; 85: 393-406.

4. Beckett C, Nalivaeva NN, Belyaev ND, Turner AJ. Nuclear signalling by membrane protein intracellular domains: the AICD enigma. Cell Signal 2012; 24: 402-409.

5. Ghosal K, Vogt DL, Liang M, Shen Y, Lamb BT, Pimplikar SW. Alzheimer's disease-like pathological features in transgenic mice expressing the APP intracellular domain. Proc Nat Acad Sci USA 2009; 106: 18367-18372.
6. Ghosal K, Stathopoulos A, Pimplikar SW. APP intracellular domain impairs adult neurogenesis in transgenic mice by inducing neuroinflammation. PLOS ONE 2010; 5 : e11866.

7. Ghosal K, Stathopoulos A, Thomas D, Phenis D, Vitek MP, Pimplikar SW. The apolipoprotein-E-mimetic COG112 protects amyloid precursor protein intracellular domainoverexpressing animals from Alzheimer's disease-like pathological features. Neurodegener dis 2013; 12: 51-58.

8. Matrone C, Luvisetto S, La Rosa LR, Tamayev R, Pignataro A, Canu N et al. Tyr682 in the Abeta-precursor protein intracellular domain regulates synaptic connectivity, cholinergic function, and cognitive performance. Aging Cell 2012; 11: 1084-1093.

9. Lombino F, Biundo F, Tamayev R, Arancio O, D'Adamio L. An intracellular threonine of amyloid-beta precursor protein mediates synaptic plasticity deficits and memory loss. PLOS ONE 2013; 8: e57120.

10. Wang $X$, Wang $Z$, Chen $Y$, Huang $X$, Hu Y, Zhang $R$ et al. FoxO mediates APP-induced AICD-dependent cell death. Cell Death Dis 2014; 5: e1233.

11. Winter J, Jung S, Keller S, Gregory RI, Diederichs S. Many roads to maturity: microRNA biogenesis pathways and their regulation. Nat Cell Biol 2009; 11: 228-234.

12. Kosik KS. The neuronal microRNA system. Nat Rev Neurosci 2006; 7: 911-920.

13. Pedersen ME, Snieckute G, Kagias K, Nehammer C, Multhaupt HA, Couchman JR et al. An epidermal microRNA regulates neuronal migration through control of the cellular glycosylation state. Science 2013; 341: 1404-1408.

14. Delay C, Mandemakers W, Hebert SS. MicroRNAs in Alzheimer's disease. Neurobiol Dis 2012; 46: 285-290.

15. Ma QH, Futagawa T, Yang WL, Jiang XD, Zeng L, Takeda $Y$ et al. A TAG1-APP signalling pathway through Fe65 negatively modulates neurogenesis. Nat Cell Biol 2008; 10: 283-294.

16. Ma QH, Bagnard D, Xiao ZC, Dawe GS. ATAG on to the neurogenic functions of APP. Cell Adhes Migr 2008; 2: 2-8.

17. Cao X, Sudhof TC. A transcriptionally [correction of transcriptively] active complex of APP with Fe65 and histone acetyltransferase Tip60. Science 2001; 293: 115-120.

18. Kimberly WT, Zheng JB, Guenette SY, Selkoe DJ. The intracellular domain of the beta-amyloid precursor protein is stabilized by Fe65 and translocates to the nucleus in a notch-like manner. J Biol Chem 2001; 276: 40288-40292.

19. Zhang W, Thevapriya S, Kim PJ, Yu WP, Shawn JeH, King Tan E et al. Amyloid precursor protein regulates neurogenesis by antagonizing miR-574-5p in the developing cerebral cortex. Nat Commun 2014; 5: 3330

20. Griffiths-Jones S, Saini HK, van Dongen S, Enright AJ. miRBase: tools for microRNA genomics. Nucleic Acid Res 2008; 36: D154-D158.

21. Monteys AM, Spengler RM, Wan J, Tecedor L, Lennox KA, Xing Y et al. Structure and activity of putative intronic miRNA promoters. RNA 2010; 16: 495-505.

22. Zhou XF, Ruan JH, Wang GD, Zhang WX. Characterization and identification of microRNA core promoters in four model species. PLos Comput Biol 2007; 3: 412-423.

23. Dweep H, Sticht C, Pandey P, Gretz N. miRWalk-database: prediction of possible miRNA binding sites by "walking" the genes of three genomes. J Biomed Inform 2011; 44: 839-847.

24. Huang daW, Sherman BT, Lempicki RA. Systematic and integrative analysis of large gene lists using DAVID bioinformatics resources. Nat Protoc 2009; 4: 44-57.

25. Belyaev ND, Nalivaeva NN, Makova NZ, Turner AJ. Neprilysin gene expression requires binding of the amyloid precursor protein intracellular domain to its promoter: implications for Alzheimer disease. EMBO Rep 2009; 10: 94-100.

26. Donato R, Miljan EA, Hines SJ, Aouabdi S, Pollock K, Patel S et al. Differential development of neuronal physiological responsiveness in two human neural stem cell lines. BMC Neurosci 2007; 8: 36

27. Rosenbloom KR, Sloan CA, Malladi VS, Dreszer TR, Learned K, Kirkup VM et al. ENCODE data in the UCSC Genome Browser: year 5 update. Nucleic Acid Res 2013; 41: D56-D63.

28. Winner B, Kohl Z, Gage FH. Neurodegenerative disease and adult neurogenesis. Eur J Neurosci 2011; 33: 1139-1151.

29. Lewis BP, Burge CB, Bartel DP. Conserved seed pairing, often flanked by adenosines, indicates that thousands of human genes are microRNA targets. Cell 2005; 120: 15-20.

30. Lim LP, Lau NC, Garrett-Engele P, Grimson A, Schelter JM, Castle J et al. Microarray analysis shows that some microRNAs downregulate large numbers of target mRNAs. Nature 2005; 433: 769-773

31. Thomson DW, Bracken CP, Goodall GJ. Experimental strategies for microRNA target identification. Nucleic Acid Res 2011; 39: 6845-6853.

32. Hu H, Li S, Cui X, Lv X, Jiao Y, Yu F et al. The overexpression of hypomethylated miR-663 induces chemotherapy resistance in human breast cancer cells by targeting heparin sulfate proteoglycan 2 (HSPG2). J Biol Chem 2013; 288: 10973-10985.

33. Yi C, Wang Q, Wang L, Huang Y, Li L, Liu L et al. MiR-663, a microRNA targeting p21(WAF1/ CIP1), promotes the proliferation and tumorigenesis of nasopharyngeal carcinoma. Oncogene 2012; 31: 4421-4433

34. Grimson A, Farh KK, Johnston WK, Garrett-Engele P, Lim LP, Bartel DP. MicroRNA targeting specificity in mammals: determinants beyond seed pairing. Mol Cell 2007; 27: 91-105.

35. Bailey TL, Boden M, Buske FA, Frith M, Grant CE, Clementi L et al. MEME SUITE: tools for motif discovery and searching. Nucleic Acid Res 2009; 37: W202-W208.

36. Alexiou P, Maragkakis M, Papadopoulos GL, Simmosis VA, Zhang L, Hatzigeorgiou AG. The DIANA-mirExTra web server: from gene expression data to microRNA function. PLoS One 2010; 5: e9171. 
37. Barbagallo AP, Wang Z, Zheng H, D'Adamio L. A single tyrosine residue in the amyloid precursor protein intracellular domain is essential for developmental function. $J$ Biol Chem 2011; 286: 8717-8721.

38. Aydin D, Weyer SW, Muller UC. Functions of the APP gene family in the nervous system: insights from mouse models. Exp Brain Res 2012; 217: 423-434

39. Gersbacher MT, Goodger ZV, Trutzel A, Bundschuh D, Nitsch RM, Konietzko U. Turnover of amyloid precursor protein family members determines their nuclear signaling capability. PLOS ONE 2013; 8: e69363.

40. Belyaev ND, Kellett KA, Beckett C, Makova NZ, Revett TJ, Nalivaeva NN et al. The transcriptionally active amyloid precursor protein (APP) intracellular domain is preferentially produced from the 695 isoform of APP in a $\{$ beta\}-secretase-dependent pathway. J Biol Chem 2010; 285: 41443-41454.

41. Saini HK, Griffiths-Jones S, Enright AJ. Genomic analysis of human microRNA transcripts. Proc Natl Acad Sci USA 2007; 104: 17719-17724.

42. Cai $\mathrm{XZ}$, Hagedorn $\mathrm{CH}$, Cullen BR. Human microRNAs are processed from capped, polyadenylated transcripts that can also function as mRNAs. RNA 2004; 10: 1957-1966.

43. Beukelaers $P$, Vandenbosch R, Caron N, Nguyen L, Belachew S, Moonen G et al. Cdk6dependent regulation of $\mathrm{G}(1)$ length controls adult neurogenesis. Stem Cells 2011; 29: 713-724.

44. Sun AX, Crabtree GR, Yoo AS. MicroRNAs: regulators of neuronal fate. Curr Opin Cell Biol 2013; 25: 215-221.

45. Shi Y, Chen C, Zhang X, Liu Q, Xu JL, Zhang HR et al. Primate-specific miR-663 functions as a tumor suppressor by targeting PIK3CD and predicts the prognosis of human glioblastoma. Clin Cancer Res 2014; 20: 1803-1813.

46. Yan-Fang $T$, Jian N, Jun L, Na W, Pei-Fang X, Wen-Li Z et al. The promoter of miR-663 is hypermethylated in Chinese pediatric acute myeloid leukemia (AML). BMC Med Genet 2013; 14: 74 .

47. Jian P, Li ZW, Fang TY, Jian W, Zhuan Z, Mei LX et al. Retinoic acid induces HL-60 cell differentiation via the upregulation of miR-663. J Hematol Oncol 2011; 4: 20

48. Tili E, Michaille JJ, Alder H, Volinia S, Delmas D, Latruffe $\mathrm{N}$ et al. Resveratrol modulates the levels of microRNAs targeting genes encoding tumor-suppressors and effectors of TGFbeta signaling pathway in SW480 cells. Biochem Pharmacol 2010; 80: 2057-2065.

49. Yang $Y$, Wang LL, Wang HX, Guo ZK, Gao XF, Cen J et al. The epigenetically-regulated miR-663 targets H-ras in K-562 cells. FEBS J 2013; 280: 5109-5117.

50. Vislovukh A, Kratassiouk G, Porto E, Gralievska N, Beldiman C, Pinna G et al Proto-oncogenic isoform A2 of eukaryotic translation elongation factor eEF1 is a target of miR-663 and miR-744. Br J Cancer 2013; 108: 2304-2311.

51. Li P, Zhu N, Yi B, Wang N, Chen M, You X et al. MicroRNA-663 Regulates Human Vascular Smooth Muscle Cell Phenotypic Switch and Vascular Neointimal Formation. Circ Res 2013; 113: 1117-1127.
52. Jin J, Cardozo T, Lovering RC, Elledge SJ, Pagano M, Harper JW. Systematic analysis and nomenclature of mammalian F-box proteins. Genes Dev 2004; 18: 2573-2580.

53. Matsumoto A, Onoyama I, Sunabori T, Kageyama R, Okano H, Nakayama KI Fbxw7-dependent degradation of Notch is required for control of "stemness" and neuronal-glial differentiation in neural stem cells. J Biol Chem 2011; 286: 13754-13764.

54. Westbrook TF, Hu G, Ang XL, Mulligan P, Pavlova NN, Liang A et al. SCFbeta-TRCP controls oncogenic transformation and neural differentiation through REST degradation. Nature 2008; 452: 370-374.

55. Mi D, Carr CB, Georgala PA, Huang YT, Manuel MN, Jeanes E et al. Pax6 exerts regional control of cortical progenitor proliferation via direct repression of Cdk6 and hypophosphorylation of pRb. Neuron 2013; 78: 269-284.

56. Kozomara A, Griffiths-Jones S. miRBase: annotating high confidence microRNAs using deep sequencing data. Nucleic Acid Res 2014; 42: D68-D73.

57. Chiang HR, Schoenfeld LW, Ruby JG, Auyeung VC, Spies N, Baek D et al. Mammalian microRNAs: experimental evaluation of novel and previously annotated genes. Genes Dev 2010; 24: 992-1009.

58. Johnson R, Teh $\mathrm{CH}$, Kunarso $\mathrm{G}$, Wong KY, Srinivasan $\mathrm{G}$, Cooper ML et al. REST regulates distinct transcriptional networks in embryonic and neural stem cells. PLOS Biol 2008; 6 e256.

59. Zhang Y, Liu T, Meyer CA, Eeckhoute J, Johnson DS, Bernstein BE et al. Model-based analysis of ChIP-Seq (MACS). Genome Biol 2008; 9: R137.

60. Sandoval J, Rodriguez JL, Tur G, Serviddio G, Pereda J, Boukaba A et al. RNAPol-ChIP: a novel application of chromatin immunoprecipitation to the analysis of real-time gene transcription. Nucleic Acid Res 2004; 32: e88.

61. Siatskas C, Seach N, Sun G, Emerson-Webber A, Silvain A, Toh BH et al. Thymic gene transfer of myelin oligodendrocyte glycoprotein ameliorates the onset but not the progression of autoimmune demyelination. Mol Ther 2012; 20: 1349-1359.

cc (i) Cell Death and Disease is an open-access journal published by Nature Publishing Group. This work is licensed under a Creative Commons Attribution 4.0 International Licence. The images or other third party material in this article are included in the article's Creative Commons licence, unless indicated otherwise in the credit line; if the material is not included under the Creative Commons licence, users will need to obtain permission from the licence holder to reproduce the material. To view a copy of this licence, visit http://creativecommons.org/licenses/by/4.0 